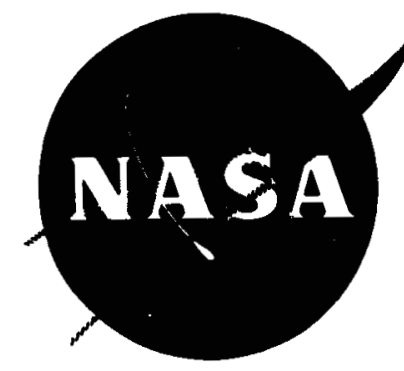

NASA TM X-52094

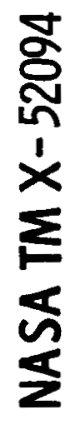
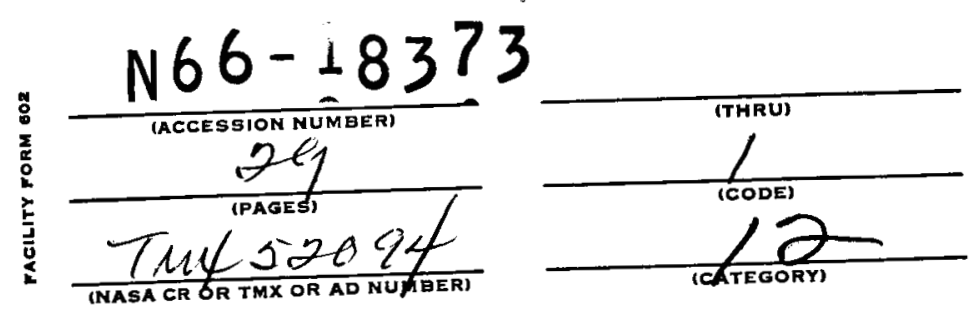

\title{
USE OF ARBITRARY QUASI-ORTHOGONALS FOR CALCULATING FLOW DISTRIBUTION IN A TURBOMACHINE
}

\section{GPO PRICE \$}

by Theodore Katsanis

Lewis Research Center

Cleveland, Ohio

CFSTI PRICE(S) \$

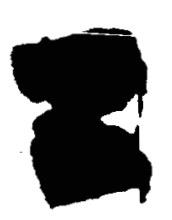

Hard copy $(\mathrm{HC})$

Microfiche (MF)

20

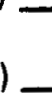

5.2

f 653 July 65

TECHNICAL PREPRINT prepared for Annual Winter Meeting of American Society of Mechanical Engineers

Chicago, Illinois, November 7-11, 1965

NATIONAL AERONAUTICS AND SPACE ADMINISTRATION - WASHINGTON, D.C. 1965 
USE OF ARBITRARY QUASI-ORTHOGONALS FOR CALCULATING FLOW DISTRIBUTION IN A TURBOMAC HINE

by Theodore Katsanis

Lewis Research Center

Cleveland, Ohio

TECHNICAL PREPRINT prepared for

Annual Winter Meeting of the

American Society of Mechanical Engineers

Chicago, Illinois, November 7-11, 1965

NATIONAL AERONAUTICS AND SPACE ADMINISTRATION 


\section{USE OF ARBITRARY QUASI-ORTHOGONALS FOR CALCULATING}

FLOW DISTRIBUTION IN A TURBOMACHINE

by Theodore Katsanis

Lewis Research Center

National Aeronautics and Space Administration

Cleveland, Ohio

\section{ABSTRACT $\quad 1737 \%$}

A method of analyzing flow through a turbomachine is summarized which is suitable for computer programming. The method, which has been reported in NASA publications, is based on an equation for the velocity gradient along an arbitrary quasi-orthogonal rather than the normal to the streamline as used in previous methods. A quasi-orthogonal is defined to be any curve that intersects every streamline between the flow boundaries exactly once, as does an orthogonal to the streamlines. With this method a streamline analysis can be made on any given stream surface. A quasi-three-dimensional solution can be obtained by using the method for a hub-to-shroud analysis, followed by blade-to-blade analyses at hub, mean, and shroud. As an example, the method was applied to a radial inflow turbine with splitter blades. The complete quasi-three-dimensional solution was obtained in a single computer run. Gulta-pr

\section{INTRODUCTION}

Quasi-three-dimensional methods have been developed and used for analyzing flow through mixed-flow turbomachines [ 1 to 4]. The first step in these methods is to obtain a two-dimensional solution on an assumed mean stream surface between the blades. This two-dimensional solution is based on an equation for the velocity gradient along the normal to the projection of the streamlines on a plane containing the axis of rotation. This plane is called the meridional plane, 
and the projections of the streamlines are called meridional streamlines. The streamlines and their normals are used to establish a grid for a meridionalplane solution. In cases where the distance between hub and shroud is great and there is a large change in flow direction within the rotor, the normals vary considerably in length and direction during the course of the calculations. Therefore, it becomes difficult to obtain a direct solution on the computer without resorting to intermediate graphical steps.

The use of normals is not essential to the method, and it appeared possible to obtain a direct solution by the use of a set of arbitrary curves from hub to shroud instead of streamline normals. These arbitrary curves will be hereinafter termed quasi-orthogonals. The quasi-orthogonals are not necessarily orthogonal to each streamline but merely intersect every streamline once across the width of the passage. The quasi-orthogonals remain fixed regardless of any change of streamlines. By using this technique, it appeared possible to develop a computer program that would calculate a streamline solution in the meridional plane without any intermediate graphical procedures even for turbomachines with wide passages and a change in direction from radial to axial within the rotor blade.

From the meridional solution it is possible to obtain blade surface velocities by several methods. One method is by means of a simple approximate formula based on the assumption of linear velocity variation between blades and absolute irrotational flow [5]. This gives good results within the rotor away from the inlet or outlet. Another method is the use of potential flow equations which may be solved by relaxation methods [6], which gives good solutions, but involve a rather tedious procedure, even with the use of a computer. It was realized 
that the basic idea used to obtain a meridional solution using quasi-orthogonals could be applied to obtain a blade-to-blade solution. In this case, the quasiorthogonals run from blade-to-blade on a stream surface determined by the meridional solution. By extending the solution upstream and downstream, a good solution throughout the rotor was obtained with a reasonable effort.

This paper summarizes a quasi-three-dimensional analysis method based on using quasi-orthogonals to obtain both the meridional and the blade-to-blade solution. Complete details are given in [7 and 8]. The numerical procedure required for obtaining solutions using a digital computer are outlined. As a numerical example of the application of the analysis method, a high specific speed radial-inlet mixed-flow gas turbine with splitter blades is analyzed. NOMENCLATURE

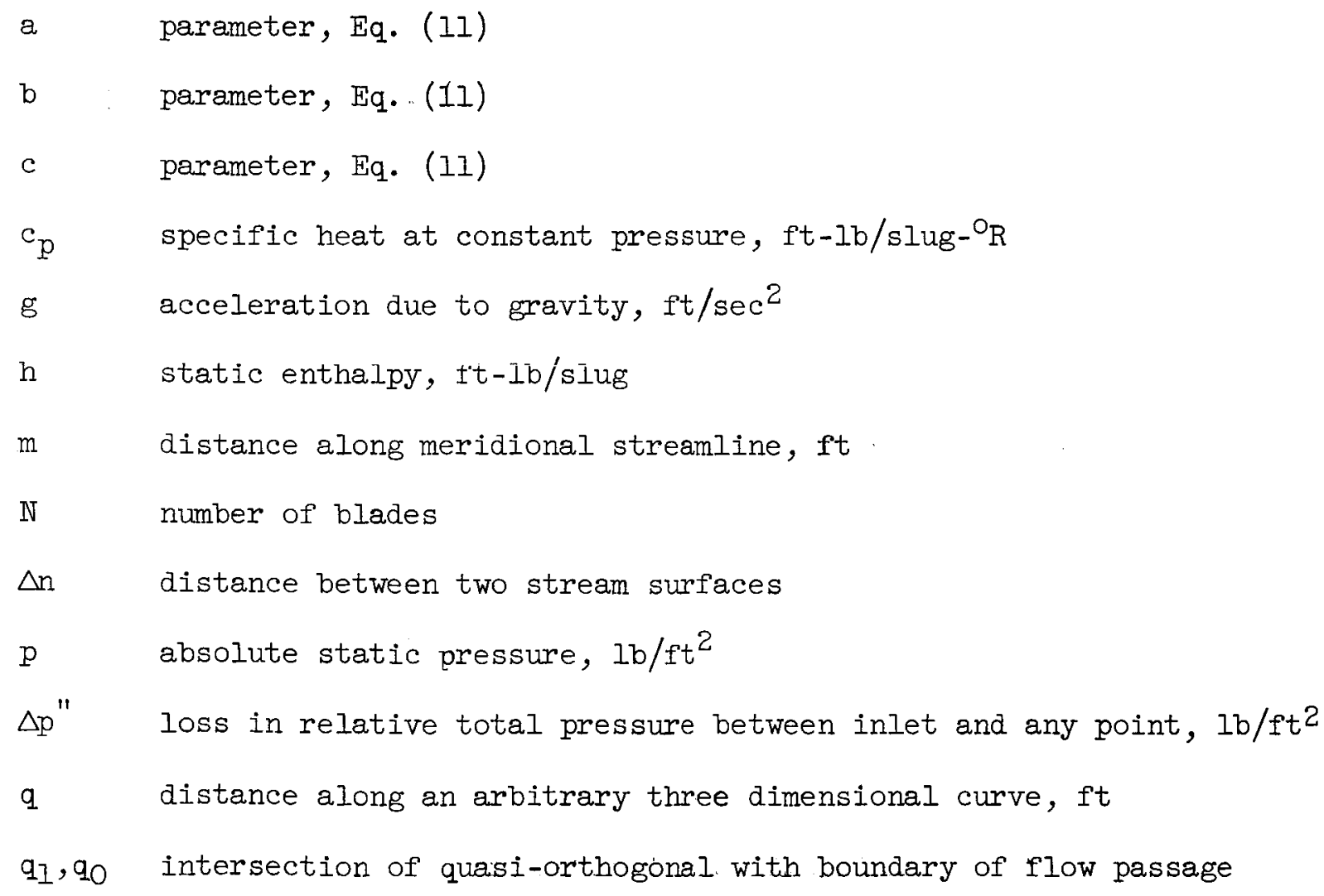


$R$ gas constant, ft-Ib/slug $-{ }^{\circ} R$

$r$ radius from axis of rotation, ft

$r_{b}$ radius where mean stream surface is assumed to deviate from mean blade shape in radial-flow turbine

$r_{c}$ radius of curvature of meridional streamline, ft

$\mathrm{T}$ temperature, ${ }^{\circ} \mathrm{R}$

$t$ time, sec

$t_{\theta}$ blade thickness in the circumferential direction, ft

$\mathrm{V}$ absolute fluid velocity, ft/sec

$\mathrm{W}$ relative fluid velocity, $\mathrm{ft} / \mathrm{sec}$

w mass flow between two stream surfaces, slug/sec

z axial coordinate, ft

$\alpha \quad$ angle between meridional streamline and $z$ axis, radians

$\beta \quad$ angle between relative velocity vector and meridional plane, radians

$r$ ratio of specific heats

$\theta$ relative angular coordinate about the axis of rotation, radians

$\lambda$ prerotation $r_{i} V_{\theta, i}$, sq $\mathrm{ft} / \mathrm{sec}$

$\rho \quad$ mass density, slug/cu ft

w rotational speed, radians/sec

Subscripts :

i inlet

$\mathrm{m}$ component is direction of meridional streamline, see Fig. 2

n component normal to quasi-orthogonal and $\Delta n$

$r \quad$ radial component

z axial component

$\theta \quad$ tangential component 
Superscripts:

absolute stagnation conditions
" relative stagnation conditions

BASIC ASSUMPTIONS AND PROCEDURE

The basic assumptions are that there is steady relative flow, and that the fluid is nonviscous and isentropic. To this a correction for losses is made by assuming a loss in relative total pressure varying fron zero at the inlet to a maximum at the outlet of the blade passage. The problem of obtaining an approximate solution to a three-dimensional fluid flow problem is considerably simplified if certain assumptions are made so that the problem can be reduced to the solution of a series of two-dimensional problems on hub-toshroud and blade-to-blade stream surfaces, as illustrated in Fig. 1. The method used herein for obtaining a streamline solution on any stream surface, whether hub-to-shroud or blade-to-blade, is essentially the same and will be hereinafter called the QO Method.

The initial assumption used here is that the mean surface between the blade is a stream surface. With this assumption, a two-dimensional meridional plane solution is obtained by the QO Method. This solution defines a blade-to-blade surface for each meridional streamline, and then solutions are obtained on these surfaces by the QO Method, giving a velocity distribution throughout the passage. EQUATIONS FOR STREAM SURFACE ANALYSIS

If a stream surface (hub-to-shroud, blade-to-blade, or other) is known or assumed, together with the streamline spacing normal to the stream surface, the problem has been reduced to a two dimensional problem. Presented here is a method of solving this two dimensional problem for fairly general cases. The 
key to the method is an equation for the directional derivative of the relative velocity $W$, which can be derived from Euler's equation for a nonviscous fluid in a rotating coordinate system:

$$
\left.\begin{array}{c}
\frac{d W_{r}}{d t}-\frac{\left(W_{\theta}+W_{r}\right)^{2}}{r}=-\frac{1}{\rho} \frac{\partial p}{\partial r} \\
\frac{d\left(r W_{\theta}+\omega r^{2}\right)}{d t}=-\frac{1}{\rho} \frac{\partial p}{\partial \theta} \\
\frac{d W_{z}}{d t}=-\frac{1}{\rho} \frac{\partial p}{\partial z}
\end{array}\right\}
$$

Here the usual convention is used that

$$
\frac{d f(r, \theta, z, t)}{d t}=\frac{d f(r(t), \theta(t), z(t), t)}{d t}
$$

where $r(t), \theta(t), z(t)$ are the parametric equations for the motion of a mass particle as a function of time. The coordinate system is shown in Fig. 2.

If we multiply Eq. ( $I$ ) by $\mathrm{Wr}, \mathrm{W}_{\theta}$, and $\mathrm{W}_{\mathrm{z}}$, respectively, then add and combine terms, we get the energy equation

$$
\frac{1}{2} \frac{d w^{2}}{d t^{2}}=\omega^{2} r \frac{d r}{d t}-\frac{1}{\rho} \frac{d p}{d t}
$$

With the assumption of steady isentropic flow, so that $\frac{1}{\rho} \frac{d p}{d t}=\frac{d h}{d t}$,

Eq. (2) can be integrated along a streamline from the inlet to obtain

$$
w^{2}-w_{i}^{2}=\omega^{2}\left(r^{2}-r_{i}^{2}\right)-2\left(h-h_{i}\right)
$$

Now, use the fact that

$$
v^{2}=w^{2}+2 v_{\theta} \omega r-\omega^{2} r^{2}
$$

to get

$$
h_{i}=h_{i}^{\prime}-\frac{v_{i}^{2}}{2}=h_{i}^{\prime}-\frac{w_{i}^{2}+2 \omega \lambda-\omega^{2} r_{i}^{2}}{2}
$$


Substituting this in Eq. (3) gives

$$
h=h_{i}-\omega \lambda+\frac{(\omega r)^{2}-W^{2}}{2}
$$

Let $q$ denote the distance along an arbitrary curve. With the assumption of isentropic flow, so that $\frac{l}{\rho} \frac{d p}{d q}=\frac{d h}{d q}$, Eq. (6) can be differentiated to obtain

$$
\frac{1}{\rho} \frac{d p}{d q}=\frac{d h_{i}^{\prime}}{d q}-\omega \frac{d \lambda}{d q}+\omega^{2} r \frac{d r}{d q}-w \frac{d W}{d q}
$$

Also the directional derivative of the pressure along this curve is

$$
\frac{d p}{d q}=\frac{\partial p}{\partial r} \frac{d r}{d q}+\frac{2 p}{d \theta} \frac{d \theta}{d q}+\frac{\partial p}{d z} \frac{d z}{d q}
$$

Evaluating the partial derivatives of the pressure in this equation by using Eq. (1), substituting this in $\mathrm{Eq}(7)$, and solving for $\frac{d W}{d q}$, gives

$$
\begin{array}{r}
\frac{d W}{d q}=\left[\frac{\omega^{2} r}{W}+\frac{1}{W} \frac{d W r}{d t}-\frac{\left(W_{\theta}+\omega r\right)^{2}}{r W}\right] \frac{d r}{d q}+\frac{1}{W} \frac{d\left(r W_{\theta}+\omega r^{2}\right)}{d t} \frac{d \theta}{d q}+\frac{1}{W} \frac{d W z}{d t} \frac{d z}{d q} \\
+\frac{1}{W} \frac{d h_{i}^{\prime}}{d q}-\frac{\omega}{W} \frac{d \lambda}{d q}
\end{array}
$$

Note that

$$
\begin{aligned}
& \mathrm{W}_{\mathrm{r}}=\mathrm{W}_{\mathrm{m}} \sin \alpha \\
& \mathrm{W}_{\mathrm{z}}=\mathrm{W}_{\mathrm{m}} \cos \alpha \\
& \mathrm{W}_{\mathrm{m}}=\mathrm{W} \cos \beta \\
& \mathrm{W}_{\theta}=\mathrm{W} \sin \beta
\end{aligned}
$$

and that

$$
\frac{d \alpha}{d t}=\frac{d \alpha}{d m} \frac{d m}{d t}=\frac{W m}{r_{c}}
$$

This can be used in evaluating the derivatives on the right side of Eq. (9) When this is done, we have

$$
\frac{d W}{d q}=a \frac{d r}{d q}+b \frac{d z}{d q}+c \frac{d \theta}{d q}+\frac{1}{W} \frac{d h_{i}^{\prime}}{d q}-\omega \frac{d \lambda}{d q}
$$


where

$$
\begin{aligned}
& \mathrm{a}=\frac{\mathrm{W} \cos \alpha \cos ^{2} \beta}{r_{\mathrm{c}}}-\frac{\mathrm{W} \sin ^{2} \beta}{r}+\sin \alpha \cos \beta \frac{d W_{m}}{d m}-2 \omega \sin \beta \\
& \mathrm{b}=-\frac{\mathrm{W} \sin \alpha \cos ^{2} \beta}{r_{c}}+\cos \alpha \cos \beta \frac{d W_{m}}{d m} \\
& c=W \sin \alpha \sin \beta \cos \beta+r \cos \beta\left(\frac{d W_{\theta}}{d m}+2 \omega \sin \alpha\right)
\end{aligned}
$$

In equation (10) the values of the parameters $h_{i}^{\prime}$ and $\lambda$ associated with a point inside the rotor is the value of that parameter at the inlet for the streamline which passes through the point. Then $\mathrm{dh} ! / \mathrm{dq}$ refers to the total enthalpy at the inlet as a function of the distance along the arbitrary curve $\mathrm{q}$ at the point considered. Further details of the derivation of Eq. (10) are given in [7].

For checking continuity of flow, it is necessary to calculate the density. Losses can be approximated by assuming a loss in relative total pressure $\Delta p$ ". With this

$$
\rho=\left(\frac{T}{T_{i}^{\prime}}\right)^{\frac{1}{(r-1)}} \rho_{i}^{\prime}-\left[\left(\frac{T_{i}}{T_{i}^{\prime}}\right)\left(\frac{T_{1}}{T^{\prime \prime}}\right]^{\frac{1}{(r-1)}} \therefore \frac{\Delta p^{\prime \prime \prime}}{R T_{i}^{\prime}}\left(\frac{T_{i}}{T^{\prime \prime}}\right)\right.
$$

where

$$
\frac{T}{T_{i}}=1-\frac{W^{2}+2 \omega \lambda-\omega^{2} r^{2}}{2 c_{p} T_{i}^{!}}
$$

and

$$
\frac{T^{\prime \prime}}{T_{i}^{\prime}}=1-\frac{2 \omega \lambda-\omega^{2} r^{2}}{2 c_{p} T_{i}^{!}}
$$

Weight flow across a quasi-orthogonal can now be computed by

$$
\mathrm{w}=\int_{\mathrm{q}_{0}}^{\mathrm{q}_{1}} \rho \mathrm{w}_{\mathrm{n}} \Delta \mathrm{ndq}
$$


where $\Delta n$ is the distance between two stream surfaces on either side of the stream surface being analyzed, $w$ is the weight flow between these surfaces per blade, and $W_{n}$ is the component of $W$ normal to the surface defined by the quasi-orthogonal and the direction in which $\Delta n$ is measured. The limits $q_{0}$ and $q_{1}$ are the intersection of the quasi-orthogonal with the boundary of the flow passage. The flow area considered is indicated in Fig. 1. NUMERICAI TECHNIQUES AND PROCEDURE

The procedure for utilizing Eq. (10) to obtain a solution on a prescribed stream surface is to estimate the parameters in Eq. (11) so that Eq. (10) can be integrated with the condition that the continuity Eq. (15) be satisfied. At each quasi-orthogonal this determines a weight flow distribution, which can be used to obtain an improved streamline pattern. This leads to a better estimate of the parameters in Eq. (11), and by iteration a flow distribution is obtained on the stream surface.

\section{Calculation of Parameters in Equation (10)}

The first step in the numerical integration of equation (10) is the numerical evaluation of the parameters $\alpha, \beta, r_{c}, \frac{d W_{m}}{d m}$, and $\frac{d W_{\theta}}{d m}$ for use in Eq. (11). In order to evaluate the parameters $\alpha, \beta$, and $r_{c}$, a streamline geometry must be established. For this, fixed lines between flow boundaries are specified on the assumed stream surfaces, at severa $\perp$ stations from inlet to outlet (for either a hub-to-shroud or a blade-to-blade stream surface). These lines are the quasi-orthogonals along which Eq. (10) will be integrated. For an initial approximation to the streamlines, each quasi-orthogonal can be divided into a number of equal spaces. The quasi-orthogonals and initıal streamline assumptions are shown in Fig. 3 for a hub-to-shroud stream surface of a radial 
inlet gas turbine. The success of the method is based on the tact that, for a reasonable assumed streamline pattern, the geometrical streamline parameters involved are not too different trom those of the final solution.

Along each assumed streamline we have $r$ as a function of $z$ at a number of points. It is desired to approximate $\frac{d r}{d z}$ and $\frac{d^{2} r}{d z^{2}}$ from these values. For any functions given at discrete points only, there are several ways a curve can be fitted through these values so as to approximate the original functions. A convenient method that has received much attention lately is the piecewise cubic, with matching first and second derivatives, usually referred to as a cubic spline function. Since for small slopes, the second derivative approximates the curvature of a function, the strain energy of a spline can be approximately minimized by minimizing $\int\left(f^{\prime \prime}(x)\right)^{2} d x$, where $f(x)$ denotes the curve described by the spline. The cubic spline has this property, as is proven in [9]. Thus the cubic spline is the mathematical expression of the shape taken by an idealized spline passing through the given points. In [9] a simple procedure is outlined for determining the spline fit curve when the coordinates of the points are given together with two arbitrary end conditions. One end condition that was used was that the second derivative at an end point is one half the second derivative at the next point. This is equivalent to bending the spline slightly beyond the last point, instead of just letting it be straight. An alternate end condition is the slope at an end point. The cubic spline function provided a simple analytical method of determining many of the parameters in the equations. The cubic spline was used to determine first and second derivatives, curvature, interpolated function values, interpolated derivatives, and for integration. 
One further point concerning the spline fit should be mentioned; that is, the approximation to an actual spline curve is dependent on the slope not being too large. Experimentally, good results are obtained if the absolute value of the slope is not greater than one. In applying this method to streamlines on a radial turbine, there is a problem since the angle may be around $-90^{\circ}$ at the inlet. This is easily overcome by rotating the coordinate axes $45^{\circ}$ so that the maximum slope is about one.

$$
\begin{aligned}
& \text { After } \frac{d r}{d z} \text { and } \frac{d^{2} r}{d z^{2}} \text { have been determined, } \alpha \text { and } \frac{1}{r_{c}} \text { are given by } \\
& \alpha=\tan ^{-1} \frac{d r}{d z} \\
& \frac{I}{r_{c}}=\frac{\frac{d^{2} r}{d z^{2}}}{\left(1+\left(\frac{d r}{d z}\right)^{2}\right)^{3 / 2}}
\end{aligned}
$$

The curvature rather than the radius of curvature is computed to avoid division by zero in case $\frac{\mathrm{d}^{2} r}{\mathrm{~d}^{2}}=0$.

The next quantity to be calculated is $\beta$. Since $\theta$ is known at each quasi-orthogonal along a streamline, the cubic spline can be used to approximate $\frac{d \theta}{d m}$. The angle $\beta$ is then calculated from

$$
\beta=\tan ^{-1}\left(r \frac{d \theta}{d m}\right)
$$

For an initial calculation, $W$ may be assumed constant throughout the rotor. Since $W m=W \cos \beta$ and $W_{\theta}=W \sin \beta$, the quantities $\frac{d W_{m}}{d m}$ and $\frac{d W_{\theta}}{d m}$ can now be approximated using the cubic spline. Now all quantities necessary for the integration of $\mathrm{Eq} .(10)$ are determined. 
Integration of Equation (10)

Given an initial value of W, Eq. (10) can be accurately integrated by means of a Runge-Kutta method. Reference [7] gives further details on the method. Since the initial value is not known, a guess of some kind must be made. This results in the velocity distribution along the quasi-orthogonal. Equations (12) to (14) can be used to compute the integrand in Eq. (15), which can then be numerically integrated using the cubic spline approximation. The computed total weight flow is then compared with the actual weight flow. If the computed weight flow is too small, the initial velocity is increased, and vice versa. Then the velocity distribution and the weight flow are recalculated. A few iterations will determine the initial velocity that will give the correct weight flow.

\section{Calculation of Improved Streamlines}

From Eq. (15) the weight flow distribution along the quasi-orthogonal can also be obtained. Along any quasi-orthogonal, inverse interpolation (by a spline approximation) can be used to determine the streamline spacing that will give equal weight flow between any two adjacent streamlines (see Fig. 4). When this is done for every quasi-orthogonal from inlet to outlet, a new estimate for the streamline pattern is obtained. However, if this streamline pattern is used for further iterations the procedure does not converge due to the fact that the streamlines become rough and uneven. This may be avoided by using only a fraction of the calculated streamline corrections, perhaps one-tenth or less. With this, the streamlines remain smooth, and a solution is reached in a single computer run. 


\section{A Quasi-Three-Dimensional Solution}

The procedure described gives a solution for any known stream surface. Since the stream surfaces are not known they must be approximated. For this reason a true three-dimensional solution is not obtained, but only what is usually referred to as a quasi-three-dimensional solution. One procedure that can be used is as follows. Assume that the mean hub-to-shroud stream surface has the same shape as the mean blade surface. A deviation from this may be specified near the inlet if there is an incidence angle. For this

initial approximation, $\Delta \mathrm{n}$ in $\mathrm{Eq} .(15)$ is equal to $\frac{2 \pi r}{\mathrm{n}}-t_{\theta}$. With these assumptions the $\mathrm{QO}$ Method is used to obtain a meridional solution. The streamlines obtained from this solution are used to define surfaces of revolution from blade-to-blade, with $\Delta \mathrm{n}$ (in Eq. (15)) for these blade-toblade surfaces determined by the streamline spacing in the meridional solution. A separate blade-to-blade solution could be obtained for each streamline; however, in most cases three blade-to-blade solutions would be adequate, one at the hub, one at the mean streamline between hub and shroud, and one at the shroud. This gives a relative velocity distribution throughout the passage. Further details of the procedure outlined above are given in $[7$ and 8$]$.

The solution could be refined by using the blade-to-blade solution to obtain a better approximation to several hub-to-shroud stream surfaces, continuing until the hub-to-shroud and blade-to-blade solutions were completely consistent. This has not been done.

\section{NUMERICAL EXAMPLE}

The method outlined has been applied to the analysis of a small radial flow turbine. The calculations were made on a digital computer. The hub-shroud 
profile and quasi-orthogonals in the meridional plane are shown in Fig. 3, together with the equally spaced streamlines used for the initial assumption. The blade has radial elements, except near the trailing edge of the splitter blade, where the taper is not the same on both sides of the blade. The pertinent data for the case analyzed is given in the following table.

DATA FOR NUMERICAI EXAMPLE

\begin{tabular}{|c|c|}
\hline Total number of blades & 22 \\
\hline Number of splitter blades & 11 \\
\hline Tip radius, $r_{i}$, in. & 3.01 \\
\hline Rotational speed, r.p.m. & 38500 \\
\hline Fluid & Argon \\
\hline Weight $f l o w, 1 b / s e c$ & .611 \\
\hline Inlet total temperature $\mathrm{T}_{i}, \mathrm{O}_{\mathrm{R}}$ & 1950 \\
\hline Absolute tangential velocity at inlet, $V_{\theta, i}$, ft/sec & 883 \\
\hline Inlet relative flow angle, $\beta_{i}$, degrees & -25.1 \\
\hline Inlet total pressure, $p_{i}^{\prime}$, psia & 12.97 \\
\hline $\begin{array}{l}\text { Loss of relative total pressure between inlet } \\
\text { and outlet, } \Delta p^{\prime \prime} \text {, psi (Assumed to vary linearly } \\
\text { along streamline.) }\end{array}$ & .25 \\
\hline
\end{tabular}

The normal blade thickness was given by means of tabulated values on a grid. Blade thickness at any given point was obtained by linear interpolation. It was assumed that $h_{i}^{\prime}$ and $\lambda$ were constant across the inlet.

At the inlet, the hub-shroud stream surface was assumed to deviate from the blade surface in order to agree with the flow direction coming into the 
rotor. Let $r_{b}$ denote the radius where the mean stream surface is assumed to deviate from the mean blade shape. Equation (13) of [5] gives an approximate equation for determining $r_{b}$, which may be written as follows:

$$
r_{b}=r_{i} e^{-0.71 \Delta \theta}
$$

The stream surface was assumed to vary cubically with $r$ between $r_{b}$ and $r_{i}$. The first step in the solution is to obtain a meridional plane solution. This solution was extended downstream of the blades, by assuming a stream surface extending downstream from the blades at an angle determined by the blade angle corrected for blockage. Twenty meridional streamlines were calculated by the QO Method. The meridional streamline pattern obtained at hub, mean, and shroud is shown in Fig. 5. This gives the coordinates for a blade-to-blade surface at the mean meridional streamline, as well as the streamline spacing on the quasi-orthogonals at hub, mean, and shroud. With this, the normal streamline spacing and the blade coordinates were calculated at each of the three surfaces, and three blade-to-blade solutions were obtained by the QO Method. The three blade-to-blade solutions were extended upstream of the inlet, because of nonuniform conditions at the inlet. The solution gives the $\theta$ and $m$ coordinates of the blade-to-blade streamlines, which are plotted in Fig. 6. The complete quasi-three-dimensional solution was obtained in a single computer run, taking 6 minutes on the IBM 7094 computer.

Figure 7 shows the blade loading on main and splitter blades at hub, mean, and shroud. The velocities are increasing over most of the blade. However, there is a large negative velocity gradient on the pressure surface at the inlet near the hub. It is even more severe at the end of the splitter blade near the shroud. This indicates the splitter should be extended further from the aero- 
dynamics point of view. However, the splitter is already longer than needed at the hub, and stress consideration may not permit making the splitter extend beyond the point where it ends on the hub. Figure 8 shows the absolute velocity distribution on the stationary shroud. Here, there are large negative velocity gradients. Of course, a negative velocity gradient cannot be eliminated entirely with the leaving absolute velocity lower than the inlet velocity, although it can be minimized by careful design. On the pressure surface the nearly linear distribution is about the best possible for avoiding flow separation, under these circumstances. However, along the shroud there is an increase, and then a greater decrease in a shorter distance. It is difficult to avoid something like this if there is to be any loading of the blades at the shroud.

This type of analysis could be very useful as a design tool, since it points up the location of possible flow separation. Modifications can be made in the geometry to improve on the velocity distributions until a good design is evolved.

\section{CONCLUDING REMARKS}

A method of analysis of turbomachines is summarized that is suitable for computer programing. The method, which has been reported in references 7 and 8 , is based on a streamline analyisis of a stream surface using quasi-orthogonals. A quasi-three-dimensional solution can be obtained by using the method first for a hub-to-shroud analysis followed by blade-to-blade analyses at hub, mean, and shroud. As an example, the method was applied to a radial inflow turbine with splitter blades. The complete quasi-three-dimensional solution was obtained in a single computer run. The FORTRAN computer program used for the numerical example may be obtained from the author by anyone who is interested in using the method. 
REFERENCES

1. Hamrick, Joseph T.; Ginsburg, Ambrose; and Osborn, Walter M.: Method of Analysis for Compressible Flow Through Mixed-Flow Centrifugal Impellers of Arbitrary Design. NACA Report 1082, 1952.

2. Smith, Kenneth J.; and Hamrick, Joseph T.: A Rapid Approximate Method for the Design of Hub Shroud Profiles of Centrifugal Impellers of Given Blade Shape. NACA TN 3399, 1955.

3. Osborn, Walter M.; Smith, Kenneth J.; and Hamrick, Joseph T.: Design and Test of Mixed-Flow Impellers. VIII - Comparison of Experimental Results for Three Impellers with Shroud Redesigned by Rapid Approximation Method. NACA RM E56L07, 1957,

4. Stockman, Norbert 0.; and Kramer, John L.: Method for Design of Pump Impellers Using a High-Speed Digital Computer. NASA TN D-1562, 1963.

5. Stanitz, John D.; and Prian, Vasily D.: A Rapid Approximate Method for Determining Velocity Distribution on Impeller Blades of Centrifugal Compressors. NACA TN 2421, 1951.

6. Kramer, J. J.: Analysis of Incompressible, Nonviscous Blade-to-Blade Flow in Rotating Blade Rows. Trans. ASME, vol. 80, no. 2, Feb. 1958, pp. $263-275$.

7. Katsanis, Theodore: Use of Arbitrary Quasi-Orthogonals for Calculating Flow Disbribution in the Meridional Plane of a Turbomachine. NASA TN D-2546, 1964.

8. Katsanis, Theodore: Use of Arbitrary Quasi-Orthogonals for Calculating Flow Distribution on a Blade-to-Blade Surface in a Turbomachine. NASA TN D-2809, 1965.

9. Walsh, J. L.; Ahlberg, J. H.; and Nilson, E. N.: Best Approximation Properties of the Spline Fit. Jour. Math. and Mech., vol. 11, no. 2, Mar. 1962, pp. 225- 


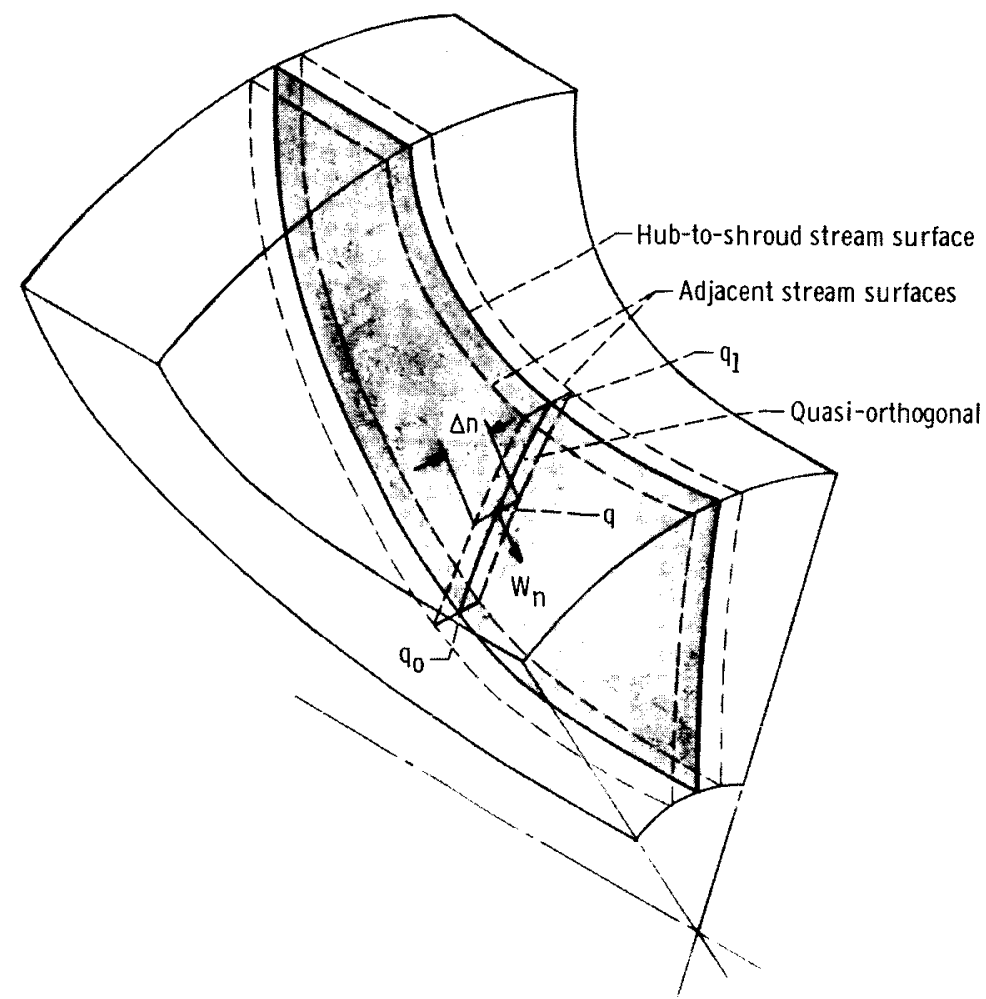

(a) Hub-to-shroud.

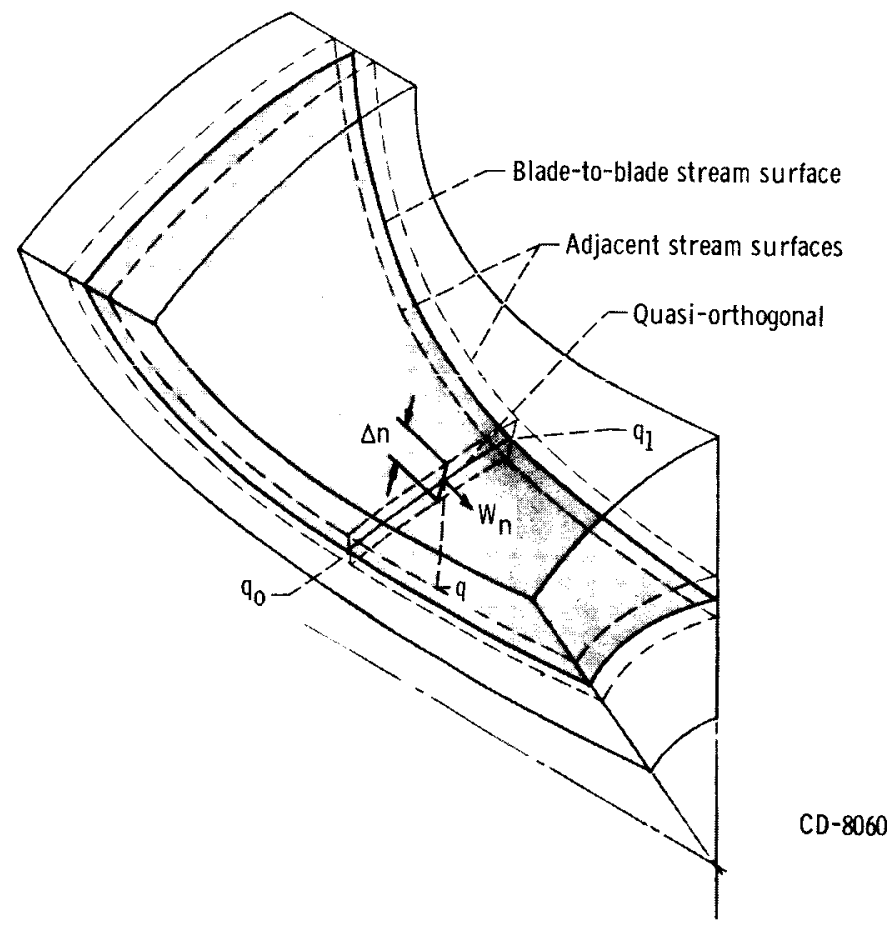

(b) Blade-to-blade.

Figure 1. - Typical stream surfaces. 


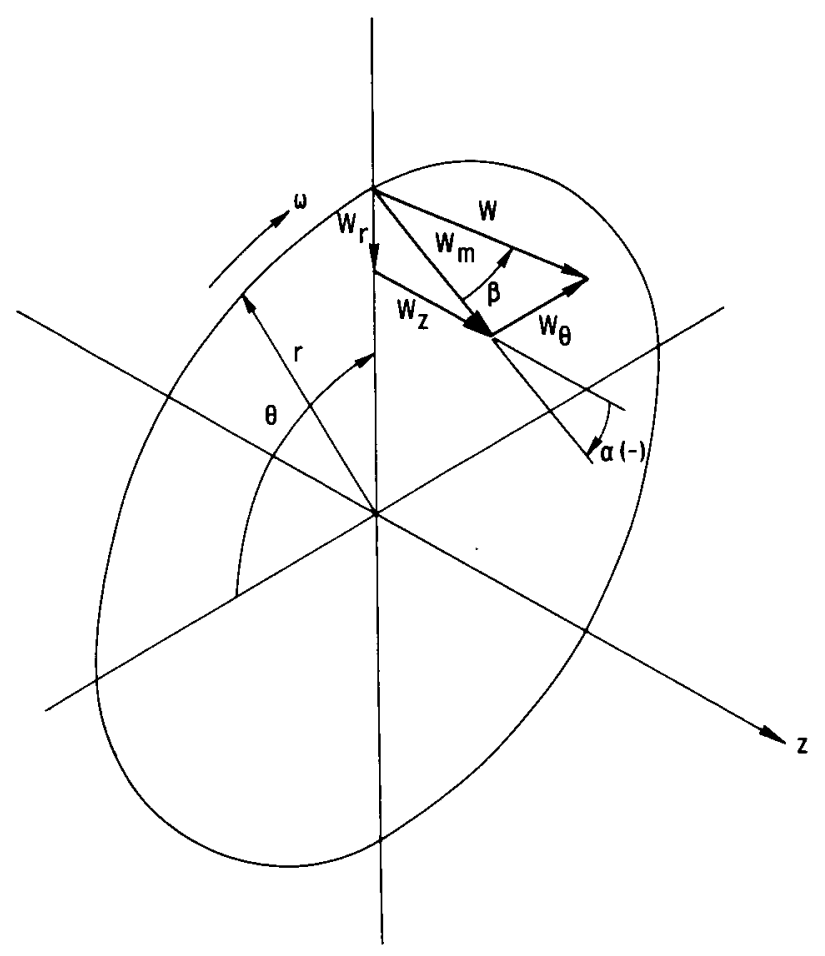

Figure 2. - Coordinate system and velocity components.

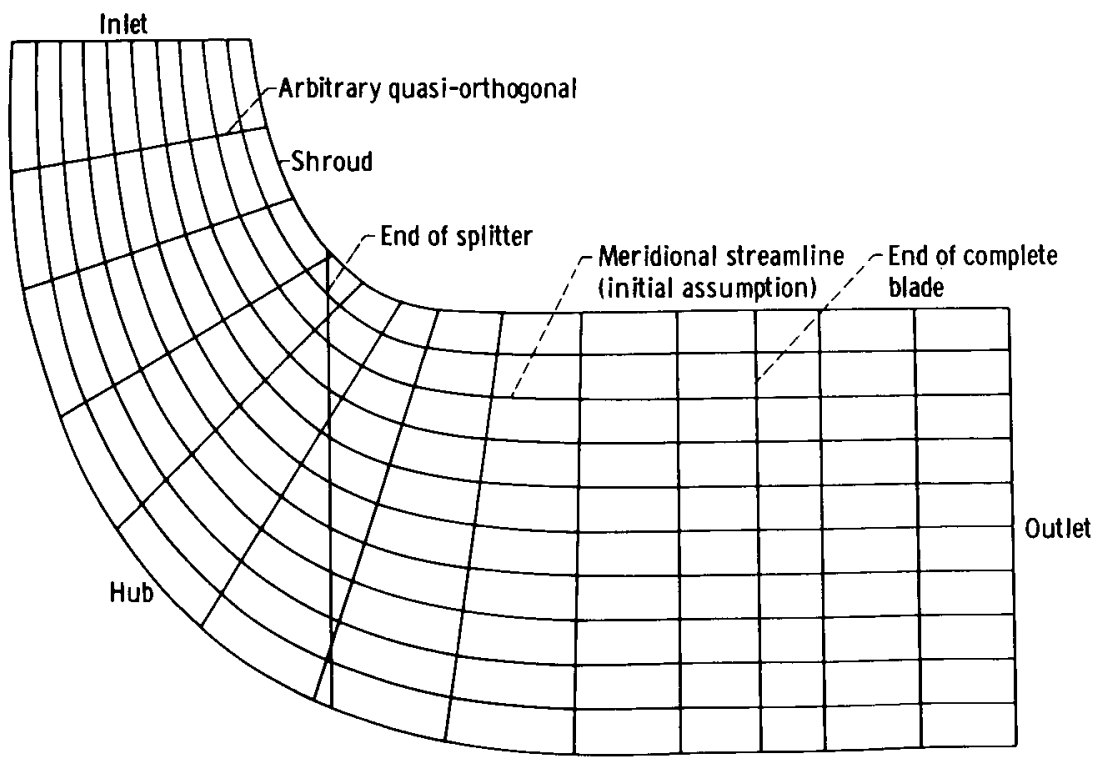

Figure 3. - Hub-shroud profile of rotor in numerical example. 


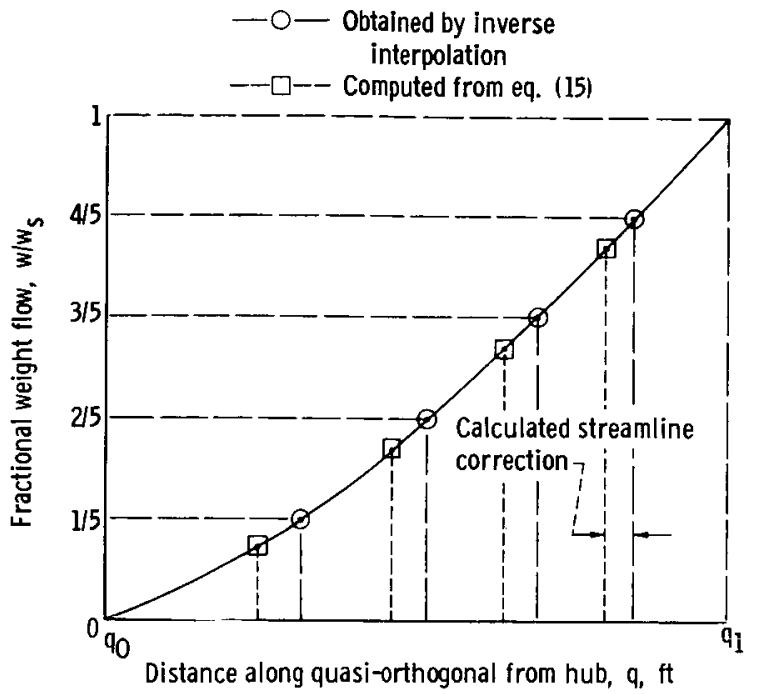

Figure 4. - Weight flow distribution along quasiorthogonal.

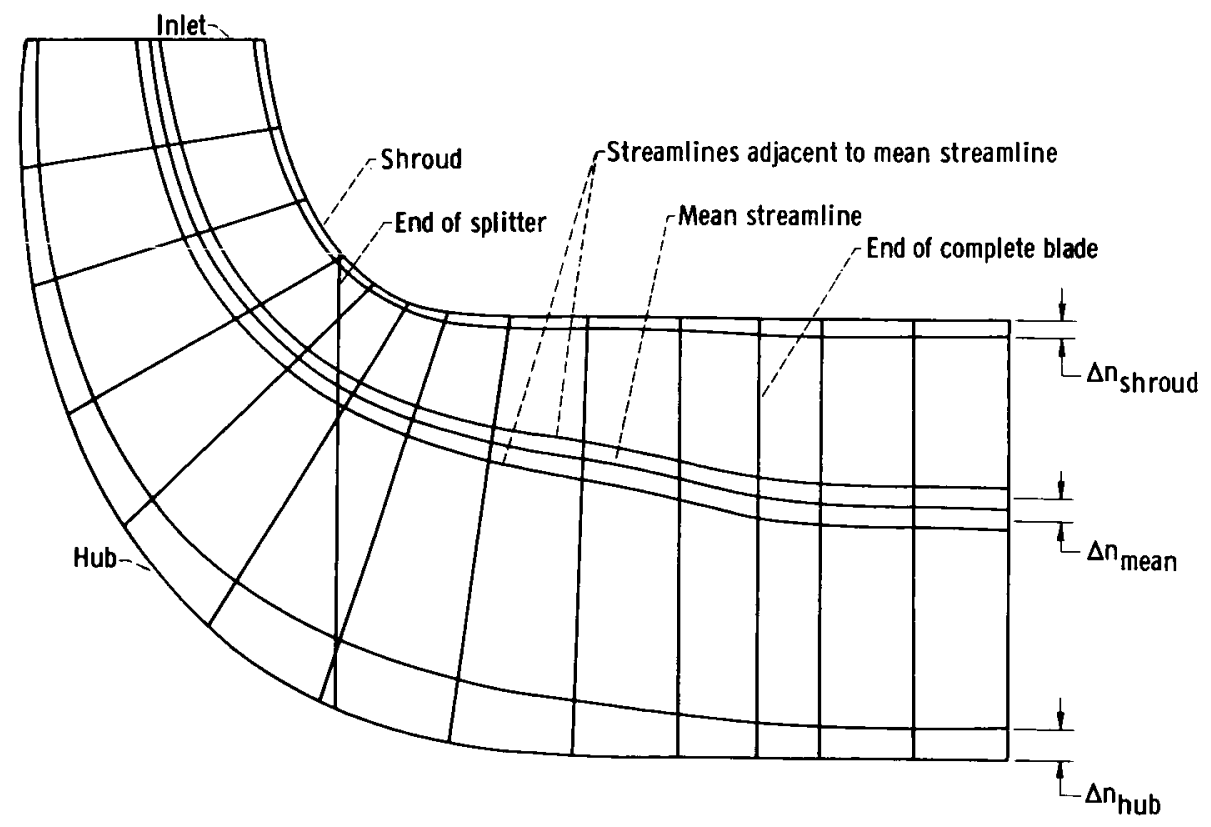

Figure 5. - Hub-shroud profile with streamlines used for blade-to-blade analysis. 


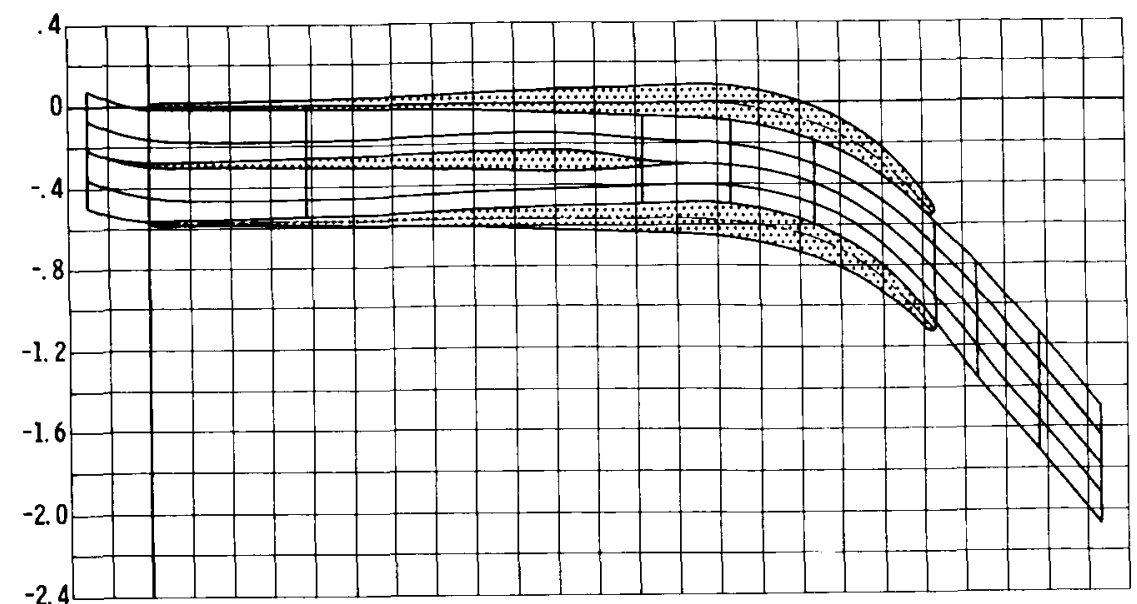

(a) Hub.

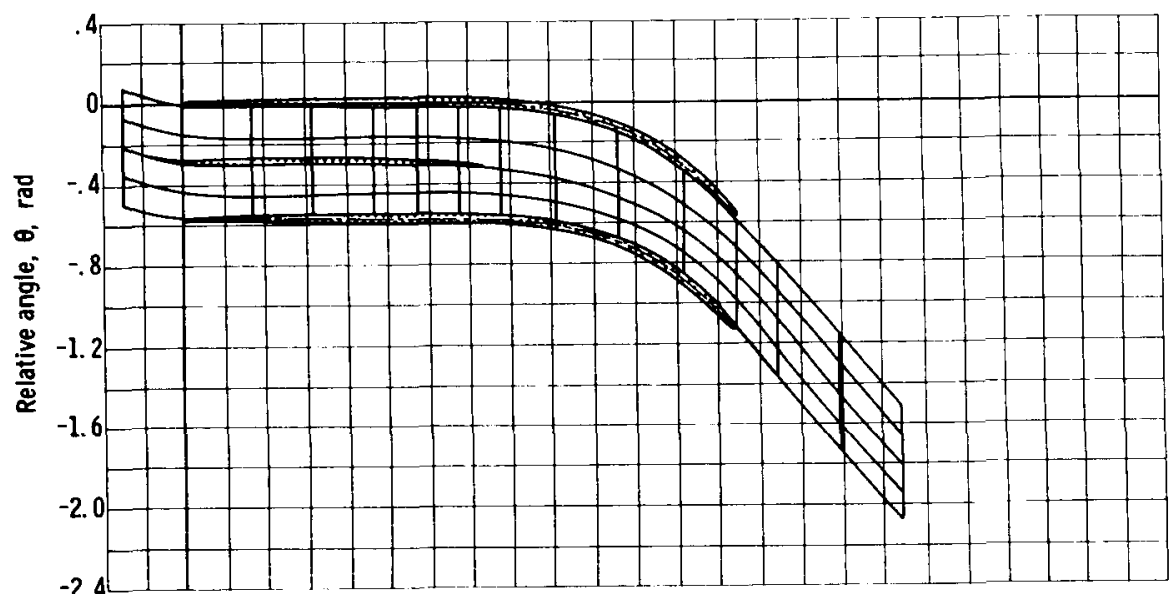

(b) Mean.

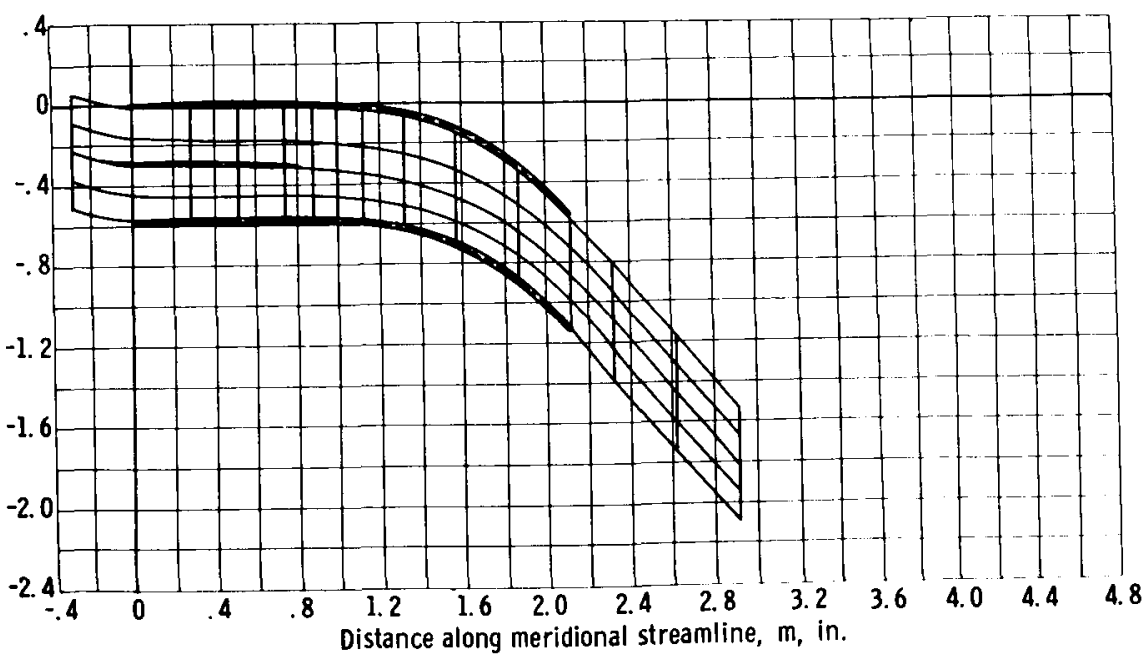

(c) Shroud.

Figure 6. - Blade-to-blade streamline pattern. 


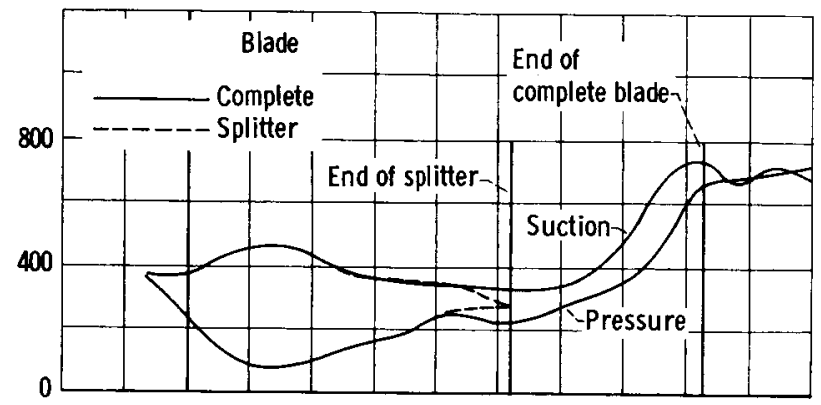

(a) Hub.

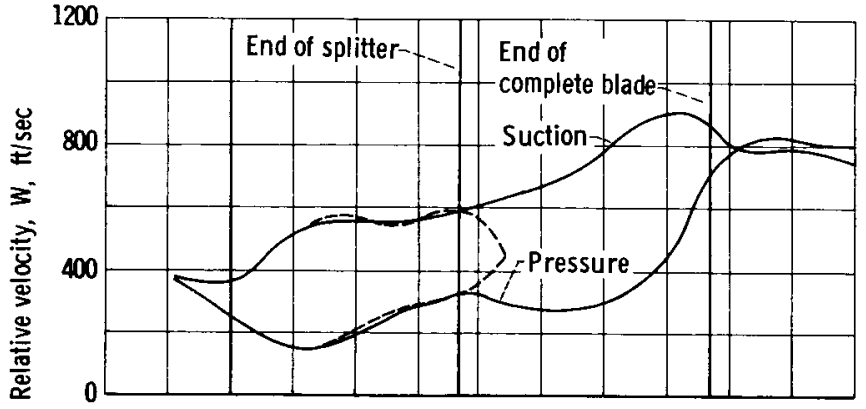

(b) Mean.

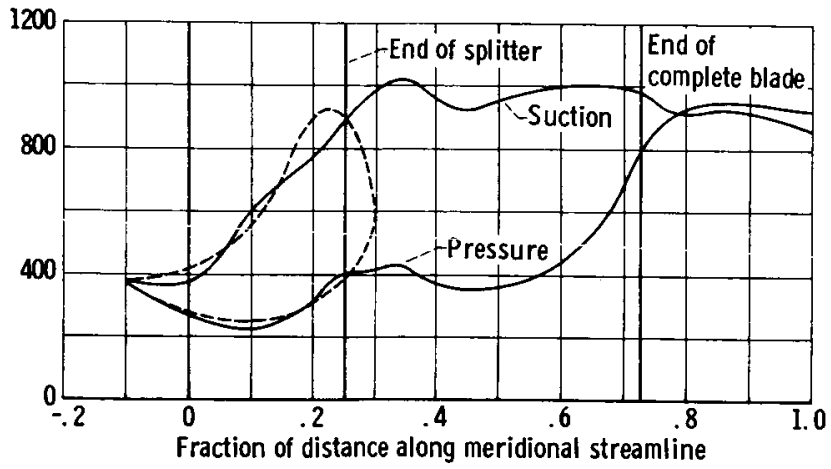

(c) Shroud.

Figure 7. - Blade velocity distribution for numerical example. 


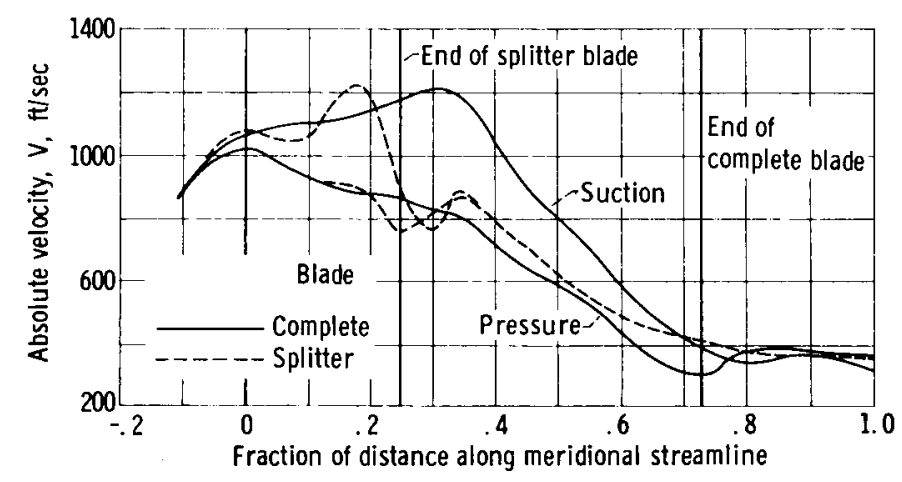

Figure 8. - Shroud absolute velocity distribution for numerical example. 